\title{
Association of apnea-hypopnea index during rapid eye movement sleep with insulin resistance in patients with suspected obstructive sleep apnea: a cross-sectional study
}

\author{
Juanjuan Zou ${ }^{1,2,3 \#}$, Yupu Liu ${ }^{1,2 \#}$, Huajun Xu ${ }^{1,2}$, Lili Meng ${ }^{1,2}$, Jianyin Zou ${ }^{1,2}$, Yingjun Qian ${ }^{1,2}$, Bin Chen ${ }^{1,2}$, \\ Hongliang Yi ${ }^{1,2}$, Jian Guan ${ }^{1,2}$, Shankai Yin ${ }^{1,2}$ \\ ${ }^{1}$ Department of Otolaryngology-Head and Neck Surgery, Shanghai Jiao Tong University Affiliated Sixth People’s Hospital, Shanghai, China; \\ ${ }^{2}$ Shanghai Key Laboratory of Sleep Disordered Breathing, Shanghai, China; ${ }^{3}$ Department of Otorhinolaryngology, Qilu Hospital of Shandong \\ University, NHC Key Laboratory of Otorhinolaryngology (Shandong University), Jinan, China \\ Contributions: (I) Conception and design: JJ Zou, H Xu, J Guan; (II) Administrative support: L Meng, JJ Zou, S Yin; (III) Provision of study materials \\ or patients: Y Qian, JY Zou, B Chen; (IV) Collection and assembly of data: Y Liu, H Yi; (V) Data analysis and interpretation: Y Liu, H Yi; (VI) \\ Manuscript writing: All authors; (VII) Final approval of manuscript: All authors. \\ "These authors contributed equally to this work. \\ Correspondence to: Jian Guan; Huajun Xu; Hongliang Yi. Otolaryngology Institute of Shanghai Jiao Tong University, Shanghai 200233, China. \\ Email: guanjian0606@sina.com; sunnydayxu2010@163.com; yihong1@126.com.
}

Background: Obstructive sleep apnea (OSA) is associated with insulin resistance. However, the association between special stages of OSA [rapid eye movement (REM) sleep] and insulin resistance is not clear. This study was designed to assess the association of the frequency of respiratory events during REM sleep with insulin resistance in adults with suspected OSA.

Methods: In this cross-sectional study, 4,062 adult participants with suspected OSA who underwent polysomnography in our sleep center between 2009 and 2016 were screened. Polysomnographic variables, biochemical indicators, and physical measurements were collected. Logistic regression analyses were conducted to determine the odds ratios (ORs) and 95\% confidence intervals (95\% CIs) for insulin resistance as assessed by the presence of hyperinsulinemia, the homeostasis model assessment of insulin resistance (HOMA-IR) index, the fasting insulin resistance index (FIRI), and Bennett's insulin sensitivity index (ISI).

Results: The final analyses included 2,899 adults with suspected OSA. Multivariate adjustments, including the apnea-hypopnea index (AHI) during non-REM sleep $\left(\mathrm{AHI}_{\mathrm{NREM}}\right)$, were performed. The AHI during REM sleep $\left(\mathrm{AHI}_{\mathrm{REM}}\right)$ was found to be independently associated with insulin resistance across increasing $\mathrm{AHI}_{\mathrm{REM}}$ quartiles. For hyperinsulinemia the ORs (95\% CIs) followed the order of $1.340(1.022,1.757), 1.210(0.882$, 1.660), and 1.632 (1.103, 2.416). For abnormal HOMA-IR, ORs (95\% CIs) were 1.287 (0.998, 1.661), 1.263 $(0.933,1.711)$, and $1.556(1.056,2.293)$. For abnormal FIRI, ORs (95\% CIs) were 1.386 (1.048, 1.835), $1.317(0.954,1.818)$, and $1.888(1.269,2.807)$. For abnormal Bennett's ISI, ORs (95\% CIs) were 1.297 $(1.003,1.678), 1.287(0.949,1.747)$, and $1.663(1.127,2.452)$. All linear trends were statistically significant $(\mathrm{P}<0.01)$. Additionally, the results showed that REM sleep duration was independently associated with hyperinsulinemia (OR =0.777, 95\% CI: 0.615-0.982; $\mathrm{P}<0.05)$.

Conclusions: $\mathrm{AHI}_{\mathrm{REM}}$ was independently associated with hyperinsulinemia and an abnormal HOMA-IR, FIRI, and Bennett's ISI in adults with suspected OSA. Additionally, REM sleep duration was independently associated with hyperinsulinemia.

Keywords: Obstructive sleep apnea (OSA); rapid eye movement sleep (REM sleep); sleep duration; insulin resistance

Submitted Apr 06, 2020. Accepted for publication Nov 08, 2020.

doi: $10.21037 /$ atm-20-3165

View this article at: http://dx.doi.org/10.21037/atm-20-3165

(c) Annals of Translational Medicine. All rights reserved. 


\section{Introduction}

Obstructive sleep apnea (OSA) is a common chronic disease that is characterized by recurrent complete or partial upper airway obstruction during sleep resulting in intermittent hypoxia, hypercapnia, and cortical microarousals (1). Accumulating evidence indicates that OSA is related to various clinical sequelae, including diabetes, hypertension, dyslipidemia, stroke, and cardiovascular events $(2,3)$. Insulin resistance has been suggested as an important shared risk factor for these sequelae $(4,5)$.

OSA can occur during rapid eye movement (REM) sleep as well as non-REM (NREM) sleep. Although REM sleep accounts for only approximately $25 \%$ of a person's total sleep period, the propensity for upper airway collapse during REM sleep is greater due to the cholinergic mediation of genioglossus muscle suppression during this stage (6). Additionally, during REM sleep, the duration of respiratory events is longer, oxygen desaturation is greater, and respiratory effort is lower $(7,8)$. Despite previous studies having uncovered an independent relationship between OSA and insulin resistance $(9,10)$, the REM sleep stage is a unique stage, and the associations of insulin resistance with respiratory events and sleep duration during this stage have not been illuminated.

OSA during REM sleep is independently associated with increased carotid intima thickness (11), poor glycemic control (12) and recurrent cardiovascular events (13); however, little is known about its association with insulin resistance. A recent study (14) found that the apneahypopnea index (AHI) during REM sleep $\left(\mathrm{AHI}_{\mathrm{REM}}\right)$ was associated with the homeostasis model assessment of insulin resistance (HOMA-IR) index. However, that study's findings were limited because only middle-aged and elderly participants were included, attended in-laboratory polysomnography (PSG) data were lacking, the risk of insulin resistance was not evaluated, and confounding factors were not fully adjusted for, including blood pressure (BP), which is closely associated with insulin resistance $(15,16)$. Studies have also shown that repeated arousal during REM sleep can lead to a decrease in REM sleep duration, which in turn influences the levels of various adipokines and the activity of the hypothalamic-pituitaryadrenal axis $(17,18)$. Thus, we speculate that REM sleep duration is related to insulin resistance.

Therefore, the primary objective of this large crosssectional study was to investigate the association between $\mathrm{AHI}_{\mathrm{REM}}$ and insulin resistance in adults of all ages using laboratory PSG data.

We present the following article in accordance with the STROBE reporting checklist (available at http://dx.doi. org/10.21037/atm-20-3165).

\section{Methods}

\section{Study population}

The data reported in this cross-sectional study were obtained from the Shanghai Sleep Health Study (SSHS) cohort, which has been previously described (19). Initially, 4,062 consecutive participants aged 18 to 88 who underwent overnight PSG for suspected OSA in the sleep center of Shanghai Jiao Tong University Affiliated Sixth People's Hospital between 2009 and 2016 were recruited. The exclusion criteria were as follows: (I) a history of treatment for OSA; (II) use of lipid-lowering drugs; (III) presence of chronic diseases, such as chronic kidney disease, psychiatric disorders, malignancy, or hyperparathyroidism; (IV) $<18$ years of age; (V) treatment with insulin or hypoglycemic agents; (VI) REM sleep duration <30 min, which would reduce the precision of $\mathrm{AHI}_{\mathrm{REM}}$ estimates (13); and (VII) missing clinical data. Finally, a total of 2,899 participants were included in this study (Figure 1). Written informed consent was obtained from all participants. This study was approved by the Internal Review Board of the Institutional Ethics Committee of Shanghai Jiao Tong University Affiliated Sixth People's Hospital [2018-KY-021(K)] and was conducted in accordance with the tenets of the Declaration of Helsinki (as revised in 2013).

\section{PSG parameters}

Overnight PSG, including electroencephalogram (EEG), electrooculogram (EOG), genioglossus electromyogram (EMG), electrocardiogram (ECG), pulse oxygen saturation $\left(\mathrm{SpO}_{2}\right)$, airflow, thoracic-abdominal movement, and body position measurements, was performed in the sleep center using the Respironics Alice 4 or 5 machine (Respironics Inc., Pittsburgh, USA). All sleep stages and respiratory events were evaluated according to the criteria of the American Academic Sleep Medicine (AASM) 2007 guidelines.

Apnea was defined as the complete cessation of airflow lasting for at least $10 \mathrm{~s}$. Hypopnea was defined as either a $\geq 50 \%$ reduction in airflow for $10 \mathrm{~s}$ or more or a $<50 \%$ but discernible reduction in airflow accompanied by either a 


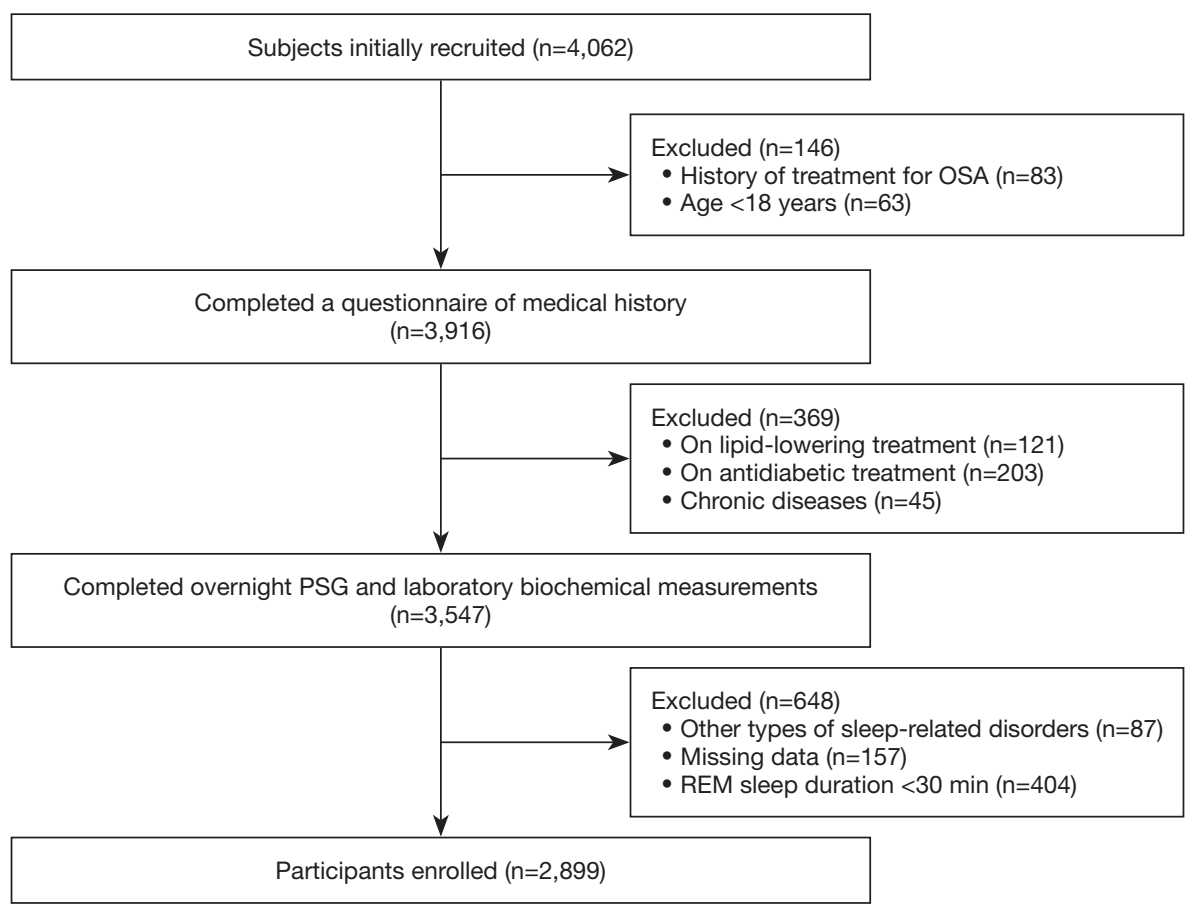

Figure 1 Flow diagram of recruitment and exclusion of participants. PSG, polysomnography.

decrease in oxyhemoglobin saturation of $\geq 4 \%$ or an arousal. The AHI was defined as the number of apnea and hypopnea events per hour during sleep. The $\mathrm{AHI}_{\mathrm{REM}}$ and $\mathrm{AHI}$ during NREM sleep $\left(\mathrm{AHI}_{\mathrm{NREM}}\right)$ were defined as the number of apnea and hypopnea events per hour during REM and NREM sleep, respectively. The arousal index was defined as the number of arousals per hour during recording. The oxygen desaturation index (ODI) was defined as the number of times the blood oxygen level decreased by $\geq 4 \%$ from baseline per hour.

\section{Biochemical measurements}

Fasting blood samples were obtained from all participants on the morning after the PSG procedure. Fasting blood glucose (FBG) levels were measured with an H-7600 autoanalyzer (Hitachi; Tokyo, Japan), and fasting insulin (FINS) levels were measured using an immunoradiology method. Four measures were used to quantify insulin resistance: (I) hyperinsulinemia; (II) HOMA-IR measured as FINS $(\mathrm{mIU} / \mathrm{L}) \times \mathrm{FBG}(\mathrm{mmol} / \mathrm{L}) / 22.5$ (20); (III) fasting insulin resistance index (FIRI) measured as FINS (mIU/L) $\times$ FBG (mmol/L)/25 (21); and (IV) Bennett's insulin sensitivity index (ISI) measured as $1 /(\ln$ FBG $\times \ln$ FINS) (22). FINS levels of $12.2 \mu \mathrm{U} / \mathrm{mL}$ or greater were defined as hyperinsulinemia.
A HOMA index of 2.5 or greater, FIRI of 2.7 or greater, and Bennett's ISI of 1.34 or greater were considered to indicate insulin resistance $(21,23,24)$.

\section{Physical measurements}

Participants' height and weight were measured according to standard procedures, with all participants wearing light clothes and barefoot. Body mass index (BMI) was calculated as the weight $(\mathrm{kg})$ divided by the height squared $\left(\mathrm{m}^{2}\right)$, and waist circumference (WC) was measured midway between the lower costal margin and iliac crest. According to the recommendations of the International Diabetes Federation (IDF) for Chinese adults, a WC of $\geq 90 \mathrm{~cm}$ in men, or $\geq 80 \mathrm{~cm}$ in women was defined as abdominal obesity (25). $\mathrm{BP}$ was recorded as the average value of three sequential measurements using standard procedures and mean arterial pressure (MAP) was calculated as (systolic $\mathrm{BP}+2 \times$ diastolic $\mathrm{BP}) / 3$.

\section{Statistical analysis}

Descriptive statistics were expressed according to $\mathrm{AHI}_{\mathrm{REM}}$ quartiles with continuous variables being presented as means \pm standard deviations (SDs) and categorical variables being 
presented as percentages. Linear trends across the $\mathrm{AHI}_{\mathrm{REM}}$ quartiles were assessed using polynomial linear trend tests for continuous variables and linear-by-linear association tests for dichotomous variables. Collinearity diagnostics were carried out to eliminate possible multicollinearity among variables. The two steps of the collinearity analyses were: (I) a preliminary analysis using Spearman's correlation; and (II) collinearity diagnostics to determine the selected covariates in the multivariate regression analyses. The detailed results are shown in Tables S1-S9. Additionally, a correlation heat map was constructed using the corrplot package in $\mathrm{R}$ software. Binary logistic regression analyses were performed to assess odds ratios (ORs) and 95\% confidence intervals (95\% CIs) for hyperinsulinemia and abnormal HOMA-IR, FIRI, and Bennet's ISI values. Several factors, including age, sex, BMI, abdominal obesity, smoking status, alcohol consumption, and MAP, were incorporated into the multivariate regression model, while $\mathrm{AHI}_{\mathrm{NREM}}$ was included when necessary. Linear trends across the four groups were computed by examining the median $\mathrm{AHI}_{\mathrm{REM}}$ values for each quartile and conducting an overall F-test. Confirmatory analysis restricted to the subgroup with $\mathrm{AHI}_{\mathrm{NREM}}$ values of $\leq 55.8$ (the highest quartile) was performed to further exclude the influence of $\mathrm{AHI}_{\text {NREM }}$.

All statistical analyses were performed with SPSS 19.0 software (SPSS, Inc.; Chicago, IL, USA) and R software. Two-tailed $\mathrm{P}$ values of $<0.05$ were considered to indicate statistical significance.

\section{Results}

\section{Baseline characteristics}

A total of 2,899 adults with suspected OSA were included in this study (Figure 1). Participant characteristics across the $\mathrm{AHI}_{\mathrm{REM}}$ quartiles $(\leq 8.1 ;>8.1, \leq 34.3 ;>34.3, \leq 56.5$; and $>56.5$ ) are presented in Table 1. On average, participants in higher $\mathrm{AHI}_{\mathrm{REM}}$ quartiles were more likely to be older, obese, and current smokers, and to have higher MAP $(\mathrm{P}<0.01$ for all linear trends). There were also linear trends between $\mathrm{AHI}_{\mathrm{REM}}$ quartiles and mean $\mathrm{SpO}_{2}$, lowest $\mathrm{SpO}_{2}$, AHI, sleep efficiency, and sleep time spent with $\mathrm{SpO}_{2}<90 \%(\mathrm{P}<0.001$ for all linear trends). Additionally, $\mathrm{AHI}_{\mathrm{REM}}$ showed positive dose-response relationships with FBG, FINS, HOMAIR, FIRI, and ISI $(\mathrm{P}<0.001$ for all linear trends). The percentages of patients with hyperinsulinemia, HOMAIR $\geq 2.5$, FIRI $\geq 2.7$, and Bennett's ISI $\leq 1.34$ rose with increasing $\mathrm{AHI}_{\mathrm{REM}}$ quartile: from $20.8 \%$ to $64.6 \%$, from
$27.4 \%$ to $72.8 \%$, from $18.1 \%$ to $63.6 \%$, and from $26.1 \%$ to $72.4 \%$, respectively ( $\mathrm{P}<0.001$ for all linear trends).

\section{Association between $A H I_{R E M}$ and insulin resistance}

Figure 2 presents a heatmap based on the Spearman correlation matrix. In the heatmap, numerical values are displayed by colors, with weaker correlations between variables displayed in light colors and stronger correlations displayed in darker colors, such as red or violet. $\mathrm{AHI}_{\mathrm{REM}}$ was found to be weakly correlated with Bennett's ISI $(r=-0.15)$, and mildly correlated with FINS ( $r=0.31)$, HOMA-IR $(\mathrm{r}=0.31)$, FIRI $(\mathrm{r}=0.31)$, hyperinsulinemia $(\mathrm{r}=0.33)$, abnormal HOMA-IR ( $\mathrm{r}=0.34)$, abnormal FIRI $(\mathrm{r}=0.35)$, and abnormal Bennett's ISI ( $\mathrm{r}=0.35$ ) (all $\mathrm{P}$ values $<0.01$ ).

After adjustment for age, sex, BMI, WC, MAP, smoking status, and alcohol consumption in Model 1 and accounting for $\mathrm{AHI}_{\mathrm{NREM}}$ in Model 2, the association between $\mathrm{AHI}_{\mathrm{REM}}$ and insulin resistance (as measured by hyperinsulinemia and abnormal HOMA-IR, FIRI, and Bennett's ISI; Table 2) was estimated using logistic regression models. In Model 1 , significant positive linear trends were observed in the ORs for insulin resistance as the $\mathrm{AHI}_{\mathrm{REM}}$ quartile increased $(\mathrm{P}<0.001$ for all linear trends). After the incorporation of $\mathrm{AHI}_{\mathrm{NREM}}$ into Model 2, the linear relationship was slightly attenuated but remained significant $(\mathrm{P}<0.01$ for all linear trends; Table 2 \& Figure $3 A, B, C, D)$.

Analysis with OSA's severities was also performed, and similar results were observed (Table S10).

\section{Subgroup verification}

Table 3 displays the characteristics of participants after exclusion of the highest $\mathrm{AHI}_{\mathrm{NREM}}$ quartile. Similar to the results of the analyses performed with all participants, on average, participants in the higher $\mathrm{AHI}_{\mathrm{REM}}$ quartiles were more obese, had higher BP and were more likely to be smokers than those in the lower quartiles $(\mathrm{P}<0.01$ for all linear trends). The sleep parameters, arousal index, ODI, and $\mathrm{AHI}$ all exhibited a linear increase across the $\mathrm{AHI}_{\mathrm{REM}}$ groups, whereas the average $\mathrm{SpO}_{2}$ and minimum $\mathrm{SpO}_{2}$ showed linear decreases $(\mathrm{P}<0.001$ for all linear trends). $\mathrm{AHI}_{\mathrm{REM}}$ showed positive dose-response relationships with FINS, HOMA-IR, FIRI, and Bennett's ISI ( $\mathrm{P}<0.001$ for all linear trends). The percentage of individuals with hyperinsulinemia and abnormal HOMA-IR, FIRI, and Bennett's ISI increased across the $\mathrm{AHI}_{\text {REM }}$ quartiles from $18.5 \%$ to $46.4 \%$, from $24.8 \%$ to $56.7 \%$, from $15.6 \%$ to 
Table 1 Characteristics, sleep parameters, and biochemical indicators of patients by $\mathrm{AHI}_{\mathrm{REM}}$ quartile

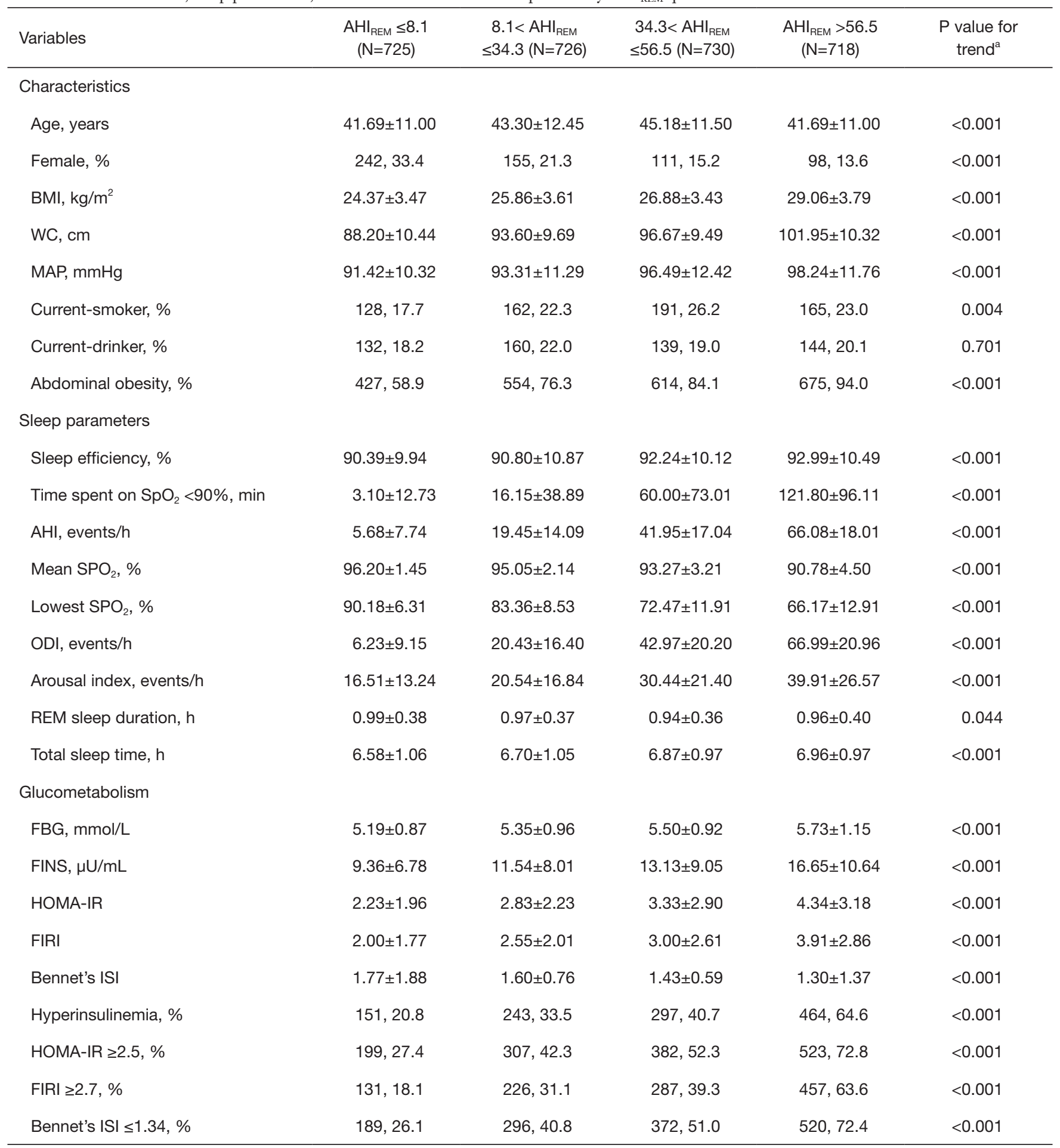

Data are presented as means \pm SD or percentage. ${ }^{a}$, tested by the polynomial linear trend test for continuous variables and the linear-bylinear association test for dichotomous variables. REM, rapid eye movement; $\mathrm{AHI}_{\mathrm{REM}}$, apnea-hypopnea index during REM sleep; $\mathrm{BMI}$, body mass index; WC, waist circumference; MAP, mean arterial pressure; AHI, apnea hypopnea index; ODI, oxygen desaturation index; FBG, fasting blood glucose; FINS, fasting insulin; HOMA-IR, homeostasis model assessment of insulin resistance; FIRI, fasting insulin resistance index; ISI, insulin sensitivity index. 


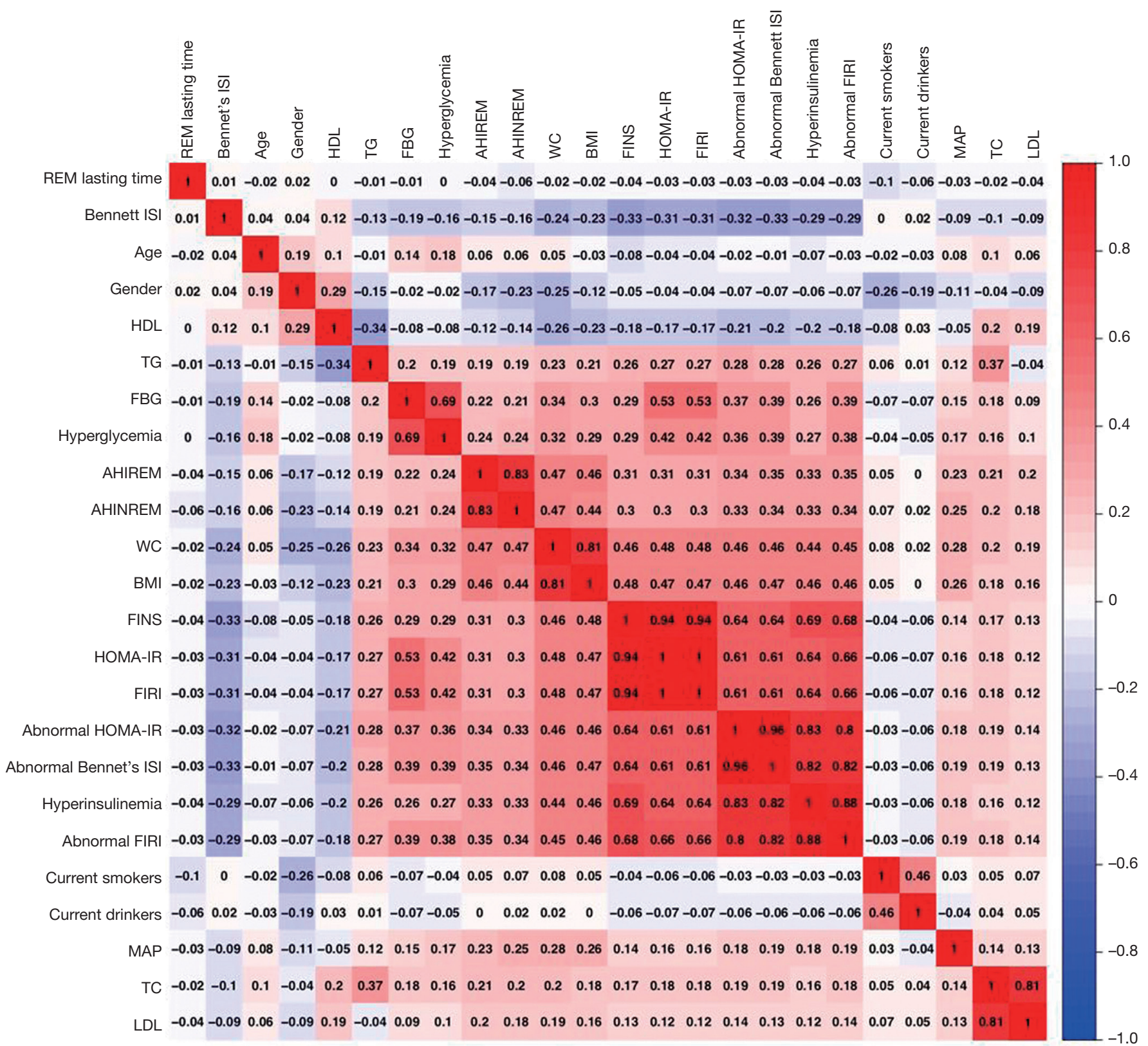

Figure 2 Heatmap based on the Spearman correlation matrix of the 24 metrics. REM, rapid eye movement; NREM, non-rapid eye movement; ISI, insulin sensitivity index; HDL, high-density lipoprotein; TG, triglyceride; FBG, fasting blood glucose; AHI, apneahypopnea index; AHIREM, AHI during REM sleep; AHINREM, AHI during NREM sleep; WC, waist circumference; BMI, body mass index; FINS, fasting insulin; HOMA-IR, homeostasis model assessment of insulin resistance; FIRI, fasting insulin resistance index; MAP, mean arterial pressure; TC, total cholesterol; LDL, low-density lipoprotein.

$44.9 \%$, and from $23.7 \%$ to $54.9 \%$, respectively $(\mathrm{P}<0.001$ for all linear trends).

Logistic regression analyses adjusted for age, sex, BMI, WC, MAP, smoking status, and alcohol consumption showed that $\mathrm{AHI}_{\mathrm{REM}}$ was independently correlated with hyperinsulinemia and abnormal HOMA-IR, FIRI, and Bennett's ISI. These correlations persisted after $\mathrm{AHI}_{\mathrm{NREM}}$ was incorporated in Model 2 (Table 4 \& Figure 3E, F, G,H). 
Table 2 Adjusted odds ratios for characteristics of insulin resistance by $\mathrm{AHI}_{\mathrm{REM}}$ quartile in Models 1 and 2

\begin{tabular}{|c|c|c|c|c|}
\hline Model & Hyperinsulinemia & HOMA-IR $\geq 2.5$ & $\mathrm{FIRI} \geq 2.7$ & Bennet's ISI $\leq 1.34$ \\
\hline $\mathrm{AHI} \mathrm{REM}_{\mathrm{REM}} \leq 8.1$ & 1 & 1 & 1 & 1 \\
\hline $8.2<\mathrm{AHI}_{\mathrm{REM}} \leq 34.3$ & $1.455(1.116,1.897)$ & $1.388(1.082,1.781)$ & $1.495(1.136,1.967)$ & $1.397(1.086,1.796)$ \\
\hline $34.3<\mathrm{AHI}_{\mathrm{REM}} \leq 56.5$ & $1.545(1.181,2.023)$ & $1.587(1.230,2.048)$ & $1.641(1.245,2.162)$ & $1.608(1.244,2.079)$ \\
\hline$P$ for trend & $<0.001$ & $<0.001$ & $<0.001$ & $<0.001$ \\
\hline \multicolumn{5}{|c|}{ Model 2 adjusted OR (95\% Cl) } \\
\hline $\mathrm{AHI}_{\mathrm{REM}} \leq 8.1$ & 1 & 1 & 1 & 1 \\
\hline $8.2<\mathrm{AHI}_{\mathrm{REM}} \leq 34.3$ & $1.340(1.022,1.757)$ & $1.287(0.998,1.661)$ & $1.386(1.048,1.835)$ & $1.297(1.003,1.678)$ \\
\hline$P$ for trend & 0.006 & 0.003 & 0.001 & 0.001 \\
\hline
\end{tabular}

Model 1: to observe the risks of insulin resistance across $\mathrm{AHI}_{\mathrm{REM}}$ quartile, the age, gender, BMI, WC, MAP, smoking status, and alcohol consumption were adjusted. Model 2: to consider the influence of $A H I_{\text {NREM }}$, we adjusted the factors in model 1 as well as $A H I_{\text {NREM. }} R E M$, rapid eye movement; NREM, non-rapid eye movement; $\mathrm{AHI}_{\mathrm{REM}}$, apnea-hypopnea index during REM sleep; $\mathrm{AHI}_{\mathrm{NREM}}$, apnea-hypopnea index during NREM sleep; HOMA-IR, homeostasis model assessment of insulin resistance; FIRI, fasting insulin resistance index; ISI, insulin sensitivity index; OR, odds ratio; $\mathrm{Cl}$, confidence interval; $\mathrm{BMI}$, body mass index; WC, waist circumference; MAP, mean arterial pressure.

\section{Association between REM sleep duration and insulin resistance}

After adjustment for age, sex, BMI, WC, MAP, AHI, smoking status, alcohol consumption, and NREM sleep duration, REM sleep duration was found to be associated with hyperinsulinemia (OR: 0.777, 95\% CI: 0.615, 0.982; Table 5). Furthermore, for every 1-hour increase in REM sleep duration, the risk of hyperinsulinemia decreased by $22.3 \%(\mathrm{P}=0.035)$.

To further confirm the results, logistic analysis was performed with adjustment for age, sex, BMI, abdominal obesity, smoking status, alcohol consumption, MAP, NREM sleep duration, $\mathrm{AHI}_{\mathrm{REM}}$, and $\mathrm{AHI}_{\mathrm{NREM}}$. REM sleep duration was found to be associated with hyperinsulinemia (OR: 0.764, 95\% CI: 0.604, 0.966; Table S11) and abnormal FIRI (OR: 0.786, 95\% CI: 0.619, 0.997; Table S11).

\section{Discussion}

This large cross-sectional study demonstrated an independent association between $\mathrm{AHI}_{\mathrm{REM}}$ and insulin resistance in adults of all ages based on laboratory PSG data and multivariate adjustments. Furthermore, a positive linear trend for the risk of insulin resistance across $\mathrm{AHI}_{\mathrm{REM}}$ quartiles was observed after adjustment for multiple variables, including $\mathrm{AHI}_{\mathrm{NREM}}$. Additionally, REM sleep duration was independently associated with the risk of hyperinsulinemia.

Several previous studies have evaluated the associations of $\mathrm{AHI}_{\mathrm{REM}}$ with various clinical sequelae, including lipid metabolism, hypertension, peripheral arterial stiffness, and cardiovascular events $(11-13,26,27)$; however, the association between $\mathrm{AHI}_{\mathrm{REM}}$ and insulin resistance, which is a common and important risk factor, has rarely been investigated. While a recent study (14) reported that $\mathrm{AHI}_{\mathrm{REM}}$ was associated with insulin resistance, this study was limited by the inclusion of only middle-aged and elderly participants; lack of in-laboratory PSG data; lack of adjustment for BP, which is closely associated with insulin resistance $(15,16)$; and lack of risk assessment of insulin resistance. The present study accounted for the above-mentioned limitations, and found that, after adjustment for age, sex, BMI, WC, MAP, smoking status, alcohol consumption, and $\mathrm{AHI}_{\mathrm{NREM}}$, patients with higher $\mathrm{AHI}_{\mathrm{REM}}$ had an increased risk of insulin resistance. The potential mechanisms involved in this association may include increased sympathetic nerve activity 
A

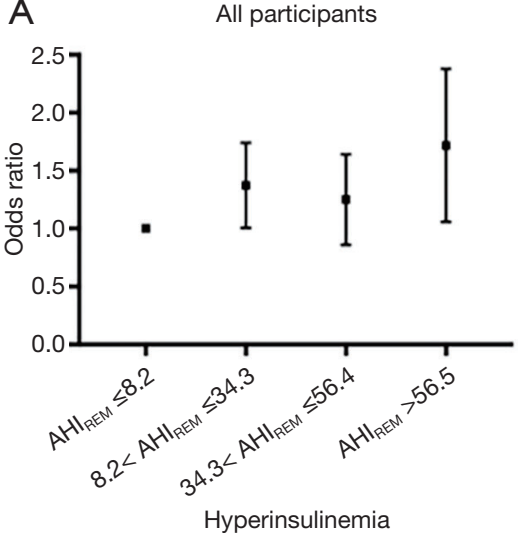

D

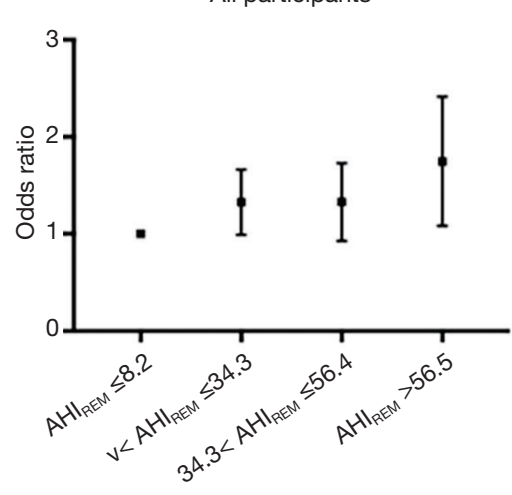

Bennet's ISI $\leq 1.34$

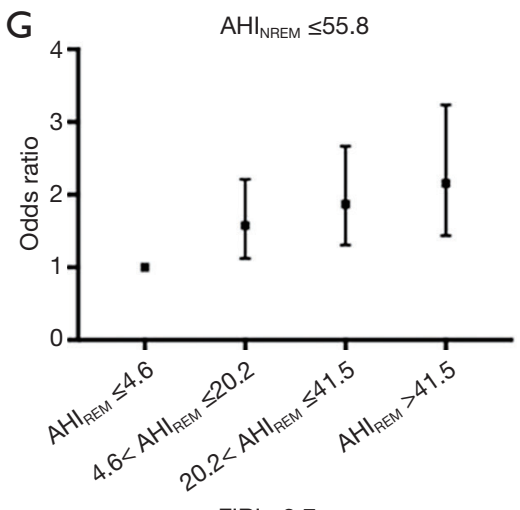

$\mathrm{FIRI} \geq 2.7$
B

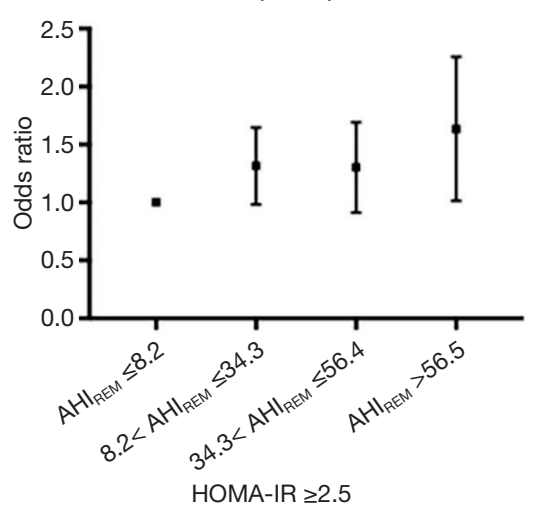

E

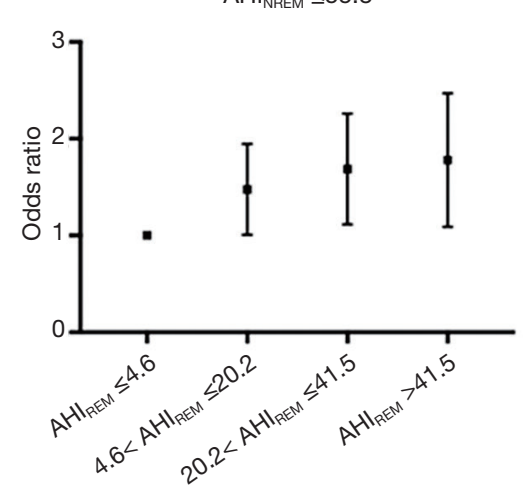

Hyperinsulinemia

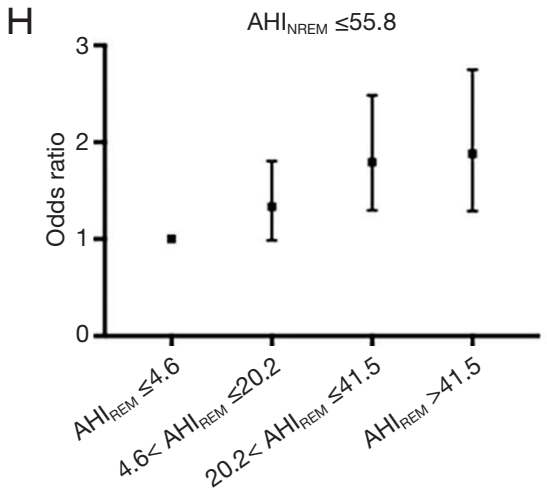

C

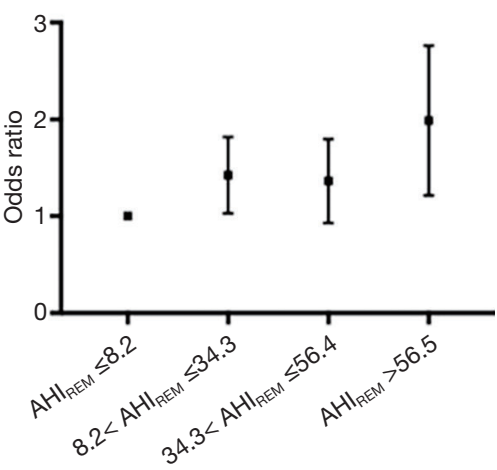

$\mathrm{FIRI} \geq 2.7$

$\mathrm{F}$

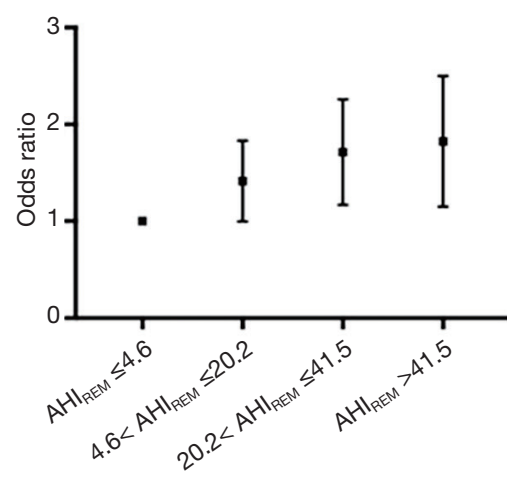

Figure 3 ORs and $95 \%$ CIs for insulin resistance across $\mathrm{AHI}_{\mathrm{REM}}$ quartiles among all participants (A,B,C,D) and subgroups (E,F,G,H). The estimates were adjusted for age, sex, BMI, WC, MAP, smoking status, alcohol consumption and $\mathrm{AHI}_{\mathrm{NREM}}$. REM, rapid eye movement; NREM, non-rapid eye movement; $\mathrm{AHI}_{\mathrm{REM}}$, apnea-hypopnea index during REM sleep; $\mathrm{AHI}_{\mathrm{NREM}}$, apnea-hypopnea index during NREM sleep; HOMA-IR, homeostasis model assessment of insulin resistance; FIRI, fasting insulin resistance index; ISI, insulin sensitivity index.

during REM sleep, decreased genioglossus muscle tone due to hypoglossal nerve inhibition and prolonged apnea and hypopnea $(7,8,28)$.
As the first-line treatment for OSA, continuous positive airway pressure (CPAP) therapy can enlarge the airway, alleviate somnolence, and improve health status (29). 
Table 3 Characteristics of subjects with $\mathrm{AHI}_{\mathrm{NREM}} \leq 55.8$ further stratified by $\mathrm{AHI}_{\mathrm{REM}}$ quartiles and with $\mathrm{AHI}_{\mathrm{NREM}}>55.8$ events/hour

\begin{tabular}{|c|c|c|c|c|c|c|}
\hline Variables & $\begin{array}{c}\mathrm{AHI}_{\mathrm{NREM}}>55.8 \\
(\mathrm{~N}=723)\end{array}$ & \multicolumn{5}{|c|}{$\mathrm{AHI}_{\mathrm{NREM}} \leq 55.8$} \\
\hline \multicolumn{7}{|l|}{ Characteristics } \\
\hline Age, years & $42.16 \pm 10.86$ & $39.01 \pm 11.97$ & $42.03 \pm 12.11$ & $44.36 \pm 12.35$ & $44.33 \pm 11.74$ & $<0.001$ \\
\hline BMI, $\mathrm{kg} / \mathrm{m}^{2}$ & $28.83 \pm 3.82$ & $24.13 \pm 3.65$ & $25.34 \pm 3.14$ & $26.24 \pm 3.65$ & $27.41 \pm 3.56$ & $<0.001$ \\
\hline WC, $\mathrm{cm}$ & $101.81 \pm 10.13$ & $87.48 \pm 10.80$ & $91.78 \pm 9.37$ & $94.56 \pm 9.61$ & $97.64 \pm 9.74$ & $<0.001$ \\
\hline MAP, mmHg & $98.44 \pm 11.83$ & $91.18 \pm 10.44$ & $92.44 \pm 10.62$ & $94.63 \pm 12.43$ & $96.44 \pm 11.75$ & $<0.001$ \\
\hline Current-smoker, \% & $184,25.4$ & $89,16.3$ & $131,23.9$ & $103,19.1$ & $139,25.6$ & 0.003 \\
\hline \multicolumn{7}{|l|}{ Sleep parameters } \\
\hline Sleep efficiency, \% & $92.90 \pm 10.37$ & $89.97 \pm 10.30$ & $91.07 \pm 10.47$ & $91.11 \pm 10.38$ & $92.53 \pm 10.27$ & $<0.001$ \\
\hline $\begin{array}{l}\text { Time spent on } \mathrm{SpO}_{2} \\
<90 \% \text {, min }\end{array}$ & $137.73 \pm 94.98$ & $2.68 \pm 12.81$ & $8.00 \pm 24.72$ & $22.96 \pm 42.43$ & $50.52 \pm 58.75$ & $<0.001$ \\
\hline AHI, times/h & $71.45 \pm 11.54$ & $4.57 \pm 7.15$ & $12.77 \pm 11.03$ & $25.34 \pm 13.03$ & $39.52 \pm 12.19$ & $<0.001$ \\
\hline Mean $\mathrm{SpO}_{2}, \%$ & $90.18 \pm 4.50$ & $96.36 \pm 1.43$ & $95.50 \pm 1.61$ & $94.68 \pm 2.33$ & $93.63 \pm 2.64$ & $<0.001$ \\
\hline Lowest $\mathrm{SpO}_{2}, \%$ & $64.63 \pm 12.71$ & $90.86 \pm 5.99$ & $86.48 \pm 7.46$ & $79.40 \pm 9.83$ & $73.30 \pm 10.95$ & $<0.001$ \\
\hline ODI, times/h & $71.67 \pm 16.57$ & $5.19 \pm 8.50$ & $13.26 \pm 12.86$ & $26.79 \pm 16.03$ & $41.29 \pm 17.39$ & $<0.001$ \\
\hline $\mathrm{FBG}, \mathrm{mmol} / \mathrm{L}$ & $5.73 \pm 1.10$ & $5.15 \pm 0.77$ & $5.27 \pm 0.88$ & $5.41 \pm 0.98$ & $5.56 \pm 1.07$ & $<0.001$ \\
\hline FINS, $\mu \mathrm{U} / \mathrm{mL}$ & $16.41 \pm 10.56$ & $8.98 \pm 6.67$ & $10.61 \pm 7.24$ & $12.04 \pm 8.22$ & $14.02 \pm 9.63$ & $<0.001$ \\
\hline HOMA-IR & $4.28 \pm 3.13$ & $2.11 \pm 1.87$ & $2.56 \pm 2.03$ & $2.99 \pm 2.44$ & $3.61 \pm 3.11$ & $<0.001$ \\
\hline FIRI & $3.85 \pm 2.82$ & $1.90 \pm 1.68$ & $2.30 \pm 1.83$ & $2.69 \pm 2.20$ & $3.25 \pm 2.80$ & $<0.001$ \\
\hline Bennet's ISI & $1.27 \pm 0.73$ & $1.83 \pm 2.13$ & $1.65 \pm 0.79$ & $1.53 \pm 0.72$ & $1.43 \pm 1.42$ & $<0.001$ \\
\hline Hyperinsulinemia, \% & $450,62.2$ & $101,18.5$ & $155,28.2$ & $197,36.5$ & $252,46.4$ & $<0.001$ \\
\hline HOMA-IR $\geq 2.5, \%$ & $516,71.4$ & $135,24.8$ & $200,36.4$ & $252,46.8$ & $308,56.7$ & $<0.001$ \\
\hline $\mathrm{FIRI} \geq 2.7, \%$ & $445,61.5$ & $85,15.6$ & $142,25.9$ & $185,34.3$ & $244,44.9$ & $<0.001$ \\
\hline Bennet's ISI $\leq 1.34, \%$ & $516,71.4$ & $129,23.7$ & $187,34.1$ & $247,45.8$ & $298,54.9$ & $<0.001$ \\
\hline
\end{tabular}

Data are presented as means \pm SD or percentage. REM, rapid eye movement; NREM, non-rapid eye movement; $A \mathrm{H}_{\mathrm{REM}}$, apnea-hypopnea index during REM sleep; $\mathrm{AHI}_{\text {NREM }}$, apnea-hypopnea index during NREM sleep; BMI, body mass index; WC, waist circumference; MAP, mean arterial pressure; ODI, oxygen desaturation index; MAI, microarousal index; FBG, fasting blood glucose; FINS, fasting insulin; HOMA-IR, homeostasis model assessment of insulin resistance; FIRI, fasting insulin resistance index; ISI, insulin sensitivity index. 
Table 4 Adjusted odds ratios for characteristics of insulin resistance by $\mathrm{AHI}_{\mathrm{REM}}$ quartile in Models 1 and 2 among subjects with $\mathrm{AHI}_{\mathrm{NREM}} \leq 55.8$

\begin{tabular}{|c|c|c|c|c|}
\hline Model & Hyperinsulinemia & HOMA-IR $\geq 2.5$ & $\mathrm{FIRI} \geq 2.7$ & Bennet's ISI $\leq 1.34$ \\
\hline $\mathrm{AHI}_{\mathrm{REM}} \leq 4.60$ & 1 & 1 & 1 & 1 \\
\hline $4.60<\mathrm{AHI}_{\mathrm{REM}} \leq 20.20$ & $1.462(1.062,2.012)$ & $1.389(1.034,1.865)$ & $1.569(1.123,2.194)$ & $1.322(0.981,1.783)$ \\
\hline $20.20<\mathrm{AHI}_{\mathrm{REM}} \leq 41.50$ & $1.733(1.260,2.385)$ & $1.708(1.270,2.298)$ & $1.845(1.323,2.574)$ & $1.752(1.299,2.362)$ \\
\hline $\mathrm{P}$ for trend & $<0.001$ & $<0.001$ & $<0.001$ & $<0.001$ \\
\hline \multicolumn{5}{|c|}{ Model 2 adjusted OR (95\% Cl) } \\
\hline $\mathrm{AHI}_{\mathrm{REM}} \leq 4.60$ & 1 & 1 & 1 & 1 \\
\hline $4.60<\mathrm{AHI}_{\mathrm{REM}} \leq 20.20$ & $1.426(1.032,1.970)$ & $1.373(1.019,1.850)$ & $1.576(1.123,2.212)$ & $1.334(0.986,1.805)$ \\
\hline$P$ for trend & 0.006 & 0.001 & $<0.001$ & $<0.001$ \\
\hline
\end{tabular}

Model 1: to observe the risks for insulin resistance across $\mathrm{AHI}_{\mathrm{REM}}$ quartile, the age, gender, $\mathrm{BMI}$, WC, MAP, smoking status, and alcohol consumption were adjusted. Model 2: to consider the influence of $A H I_{\text {NREM }}$, we adjusted the factors in model 1 as well as $A H I_{\text {NREM. }} R E M$, rapid eye movement; NREM, non-rapid eye movement; $\mathrm{AHI}_{\mathrm{REM}}$, apnea-hypopnea index during REM sleep; $\mathrm{AHI}_{\mathrm{NREM}}$, apnea-hypopnea index during NREM sleep; HOMA-IR, homeostasis model assessment of insulin resistance; FIRI, fasting insulin resistance index; ISI, insulin sensitivity index; $\mathrm{OR}$, odds ratio; $\mathrm{Cl}$, confidence interval; $\mathrm{BMI}$, body mass index; WC, waist circumference; MAP, mean arterial pressure.

However, no consensus has been reached as to whether this therapy can improve insulin resistance. Martinez and colleagues (30) reviewed related research and found that CPAP improved insulin levels and insulin resistance, whereas Jullian and colleagues (31) failed to find these beneficial effects. As REM sleep occurs mainly during the latter half of sleep, 4 hours of CPAP therapy per night may not cover all periods of REM sleep. Additionally, in the present study, $\mathrm{AHI}_{\text {REM }}$ was independently associated with insulin resistance. Therefore, it is plausible that untreated OSA during REM sleep could weaken the effects of CPAP on insulin resistance. In contrast, 7 hours of CPAP therapy per night covers more than $85 \%$ of REM sleep (12). Therefore, insufficient CPAP use may be the reason for the poor treatment effects, suggesting that more attention should be paid to verifying whether nightly CPAP treatment should be extended beyond 4 hours. In addition, higher CPAP pressure during REM sleep may be effective in thoroughly eliminating respiratory events.

In terms of sleep duration, short sleep duration is reported to be significantly associated with decreased insulin sensitivity (32), as well as increased subclinical atherosclerosis risk (33). However, to date, no studies have investigated the association between REM sleep duration and insulin resistance in OSA patients. The results of the current study showed that after multivariate adjustments, the risk of hyperinsulinemia decreased by $22.3 \%$ for every 1-hour increase in REM sleep duration. To the best of our knowledge, this is the first clinical research study to investigate the association between REM sleep duration and insulin resistance among OSA adults. Several factors may be involved in the mechanisms that mediate the association between REM sleep duration and hyperinsulinemia. Firstly, decreased REM sleep duration is related to changes in adipokine levels, which can disrupt insulin levels (34). Visfatin, for instance, competitively inhibits the binding of insulin to the insulin receptor (35). Secondly, decreased REM sleep duration results in uncontrolled negative hypothalamic-pituitary-adrenal axis feedback, and excessive levels of cortisol induce pancreatic islet $\beta$ cells to produce more insulin $(17,18)$. Thus, the present results indicate that improved sleep quality may contribute to lowering the risk of hyperinsulinemia.

The present study has several strengths, including its large sample size, assessments of standard in-laboratory PSG data, employment of multivariate adjustments, and inclusion of adults of all ages. However, there are several limitations that should be noted. Firstly, this was a cross- 


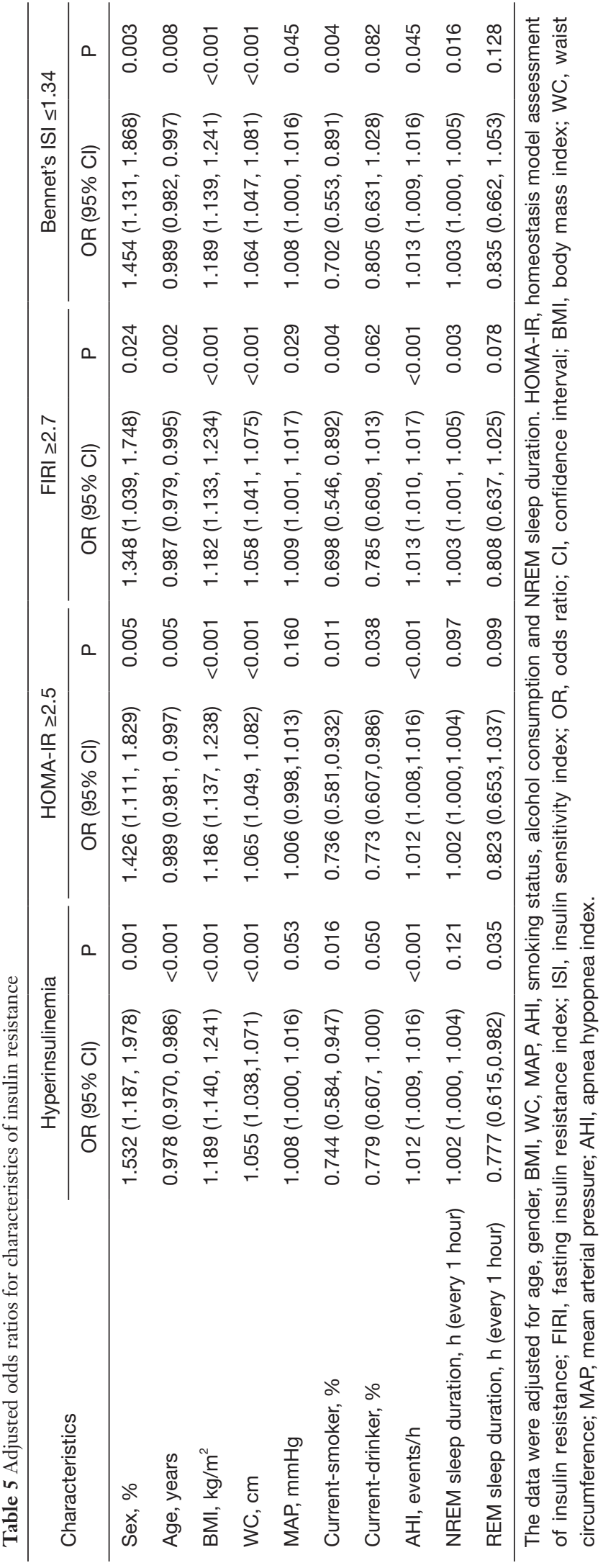

sectional study, so causality could not be assessed. Secondly, this was a hospital-based observational study rather than a community-based, prospective study. Thirdly, the participants' dietary, lifestyle, and physical exercise habits, all of which may affect insulin resistance, were not assessed. Thus, not all potentially confounding factors were fully controlled. Finally, certain data, such as the hemogloblinA1c (HbA1c) levels, were unavailable, meaning the level of insulin resistance could not be fully evaluated.

The independent associations of $\mathrm{AHI}_{\mathrm{REM}}$ or REM sleep duration with insulin resistance evidenced by this study are of great clinical significance. For instance, reducing respiratory events during REM sleep and improving sleep architecture may be beneficial for reducing the risk of insulin resistance. In other words, more attention should be devoted to increasing CPAP pressure during REM sleep or extending the nightly duration of CPAP treatment. Drugs that improve sleep quality may also be useful, especially for individuals with insulin resistance.

\section{Conclusions}

In summary, the present study demonstrated an independent association between the frequency of respiratory events during REM sleep and insulin resistance, as assessed by the presence of hyperinsulinemia, abnormal HOMAIR, FIRI, and Bennett's ISI, in adults with suspected OSA. Additionally, REM sleep duration was found to be associated with hyperinsulinemia. These findings suggest that improvement of sleep architecture and sleep quality may be beneficial in reducing the risk of insulin resistance.

\section{Acknowledgments}

We thank all of the participants and survey staffs for their participation.

Funding: This study was funded by National Key R\&D Program of China (2017YFC0112500), National Natural Science Foundation of China (81770987, 81700896, 81701306, 81770988), Shanghai Municipal Commission of Science and Technology (18DZ2260200), Shanghai Shenkang Hospital Development Center (16CR3103B), Innovation Program of Shanghai Municipal Education Commission (2017-01-07-00-02-E00047), multicenter clinical research project from school of medicine, Shanghai Jiao Tong University (DLY201502) and Shanghai Shen-Kang Hospital Management Center Project (SHDC12015101). 


\section{Footnote}

Reporting Checklist: The authors have completed the STROBE reporting checklist. Available at http://dx.doi. org/10.21037/atm-20-3165

Data Sharing Statement: Available at http://dx.doi. org/10.21037/atm-20-3165

Conflicts of Interest: All authors have completed the ICMJE uniform disclosure form (available at http://dx.doi. org/10.21037/atm-20-3165). The authors have no conflicts of interest to declare.

Ethical Statement: The authors are accountable for all aspects of the work in ensuring that questions related to the accuracy or integrity of any part of the work are appropriately investigated and resolved. This study was conducted in accordance with the tenets of the Declaration of Helsinki (as revised in 2013) and was approved by the Internal Review Board of the Institutional Ethics Committee of Shanghai Jiao Tong University Affiliated Sixth People's Hospital [2018-KY-021(K)] and informed consent was taken from all the patients.

Open Access Statement: This is an Open Access article distributed in accordance with the Creative Commons Attribution-NonCommercial-NoDerivs 4.0 International License (CC BY-NC-ND 4.0), which permits the noncommercial replication and distribution of the article with the strict proviso that no changes or edits are made and the original work is properly cited (including links to both the formal publication through the relevant DOI and the license). See: https://creativecommons.org/licenses/by-nc-nd/4.0/.

\section{References}

1. Young T, Palta M, Dempsey J, et al. The occurrence of sleep-disordered breathing among middle-aged adults. N Engl J Med 1993;328:1230-5.

2. Fu Y, Xia Y, Yi H, et al. Meta-analysis of all-cause and cardiovascular mortality in obstructive sleep apnea with or without continuous positive airway pressure treatment. Sleep Breath 2017;21:181-9.

3. Framnes SN, Arble DM. The Bidirectional Relationship Between Obstructive Sleep Apnea and Metabolic Disease. Front Endocrinol (Lausanne) 2018;9:440.

4. Ormazabal V, Nair S, Elfeky O, et al. Association between insulin resistance and the development of cardiovascular disease. Cardiovasc Diabetol 2018;17:122.

5. Deng XL, Liu Z, Wang C, et al. Insulin resistance in ischemic stroke. Metab Brain Dis 2017;32:1323-34.

6. McSharry DG, Saboisky JP, DeYoung P, et al. Physiological Mechanisms of Upper Airway Hypotonia during REM Sleep. Sleep 2014;37:561-9.

7. Findley LJ, Wilhoit SC, Suratt PM. Apnea Duration and Hypoxemia During REM Sleep in Patients with Obstructive Sleep Apnea. Chest 1985;87:432-6.

8. Krieger J, Sforza E, Boudewijns A, et al. Respiratory Effort During Obstructive Sleep Apnea. Chest 1997;112:875-84.

9. Zou J, Xia Y, Xu H, et al. Independent relationships between cardinal features of obstructive sleep apnea and glycometabolism: a cross-sectional study. Metabolism 2018;85:340-7.

10. Murphy AM, Thomas A, Crinion SJ, et al. Intermittent hypoxia in obstructive sleep apnoea mediates insulin resistance through adipose tissue inflammation. Eur Respir J 2017;49:1601731.

11. Ljunggren M, Lindberg E, Franklin KA, et al. Obstructive sleep apnea during rapid eye movement sleep is associated with early signs of atherosclerosis in women. Sleep 2018;41. doi: 10.1093/sleep/zsy099.

12. Grimaldi D, Beccuti G, Touma C, et al. Association of obstructive sleep apnea in rapid eye movement sleep with reduced glycemic control in type 2 diabetes: therapeutic implications. Diabetes Care 2014;37:355-63.

13. Aurora RN, Crainiceanu C, Gottlieb DJ, et al. Obstructive Sleep Apnea during REM Sleep and Cardiovascular Disease. Am J Respir Crit Care Med 2018;197:653-60.

14. Chami HA, Gottlieb DJ, Redline S, et al. Association between Glucose Metabolism and Sleep-disordered Breathing during REM Sleep. Am J Respir Crit Care Med 2015;192:1118-26.

15. Gluvic Z, Zaric B, Resanovic I, et al. Link between Metabolic Syndrome and Insulin Resistance. Curr Vasc Pharmacol 2017;15:30-9.

16. Jung CH, Jung SH, Lee B, et al. Relationship among age, insulin resistance, and blood pressure. J Am Soc Hypertens 2017;11:359-365.e2.

17. Van Cauter E, Leproult R, Plat L. Age-related changes in slow wave sleep and REM sleep and relationship with growth hormone and cortisol levels in healthy men. JAMA 2000;284:861-8.

18. Venancio DP, Suchecki D. Prolonged REM sleep restriction induces metabolic syndrome-related changes: Mediation by pro-inflammatory cytokines. Brain Behav 
Immun 2015;47:109-17.

19. Guan J, Yi H, Zou J, et al. Distinct severity stages of obstructive sleep apnoea are correlated with unique dyslipidaemia: large-scale observational study. Thorax 2016;71:347-55.

20. Matthews DR, Hosker JP, Rudenski AS, et al. Homeostasis model assessment: insulin resistance and beta-cell function from fasting plasma glucose and insulin concentrations in man. Diabetologia 1985;28:412-9.

21. Duncan $M H$, Singh BM, Wise PH, et al. A simple measure of insulin resistance. Lancet 1995;346:120-1.

22. Anderson RL, Hamman RF, Savage PJ, et al. Exploration of simple insulin sensitivity measures derived from frequently sampled intravenous glucose tolerance (FSIGT) tests. The Insulin Resistance Atherosclerosis Study. Am J Epidemiol 1995;142:724-32.

23. McAuley KA, Williams SM, Mann JI, et al. Diagnosing insulin resistance in the general population. Diabetes Care 2001;24:460-4.

24. Suskin N, McKelvie RS, Burns RJ, et al. Glucose and insulin abnormalities relate to functional capacity in patients with congestive heart failure. Eur Heart J 2000;21:1368-75.

25. Alberti KG, Zimmet P, Shaw J. The metabolic syndrome-a new worldwide definition. Lancet 2005;366:1059-62.

26. Xu H, Xia Y, Li X, et al. Association between obstructive sleep apnea and lipid metabolism during REM and NREM sleep. J Clin Sleep Med 2020;16:475-82.

27. Uchida T, Nishimura A, Kasai T, et al. Relationship between obstructive sleep apnoea during rapid eye movement sleep and metabolic syndrome parameters in

Cite this article as: Zou J, Liu Y, Xu H, Meng L, Zou J, Qian Y, Chen B, Yi H, Guan J, Yin S. Association of apneahypopnea index during rapid eye movement sleep with insulin resistance in patients with suspected obstructive sleep apnea: a cross-sectional study. Ann Transl Med 2021;9(3):243. doi: 10.21037/atm-20-3165 patients with type 2 diabetes mellitus. Sleep Breath 2020. [Epub ahead of print]. doi:10.1007/s11325-020-02129-7.

28. Somers VK, Dyken ME, Clary MP, et al. Sympathetic neural mechanisms in obstructive sleep apnea. J Clin Invest 1995;96:1897-904.

29. Mehrtash M, Bakker JP, Ayas N. Predictors of Continuous Positive Airway Pressure Adherence in Patients with Obstructive Sleep Apnea. Lung 2019;197:115-21.

30. Martínez-Ceron E, Fernandez-Navarro I, Garcia-Rio F. Effects of continuous positive airway pressure treatment on glucose metabolism in patients with obstructive sleep apnea. Sleep Med Rev 2016;25:121-30.

31. Jullian-Desayes I, Joyeux-Faure M, Tamisier R, et al. Impact of obstructive sleep apnea treatment by continuous positive airway pressure on cardiometabolic biomarkers: a systematic review from sham CPAP randomized controlled trials. Sleep Med Rev 2015;21:23-38.

32. van Dijk D, Balkau B, Segrestin B, et al. Associations between sleep duration and sleep debt with insulin sensitivity and insulin secretion in the EGIR-RISC Study. Diabetes Metab 2019;45:375-81.

33. Domínguez F, Fuster V, Fernández-Alvira JM, et al. Association of Sleep Duration and Quality With Subclinical Atherosclerosis. J Am Coll Cardiol 2019;73:134-44.

34. Olson CA, Hamilton NA, Somers VK. Percentage of REM sleep is associated with overnight change in leptin. J Sleep Res 2016;25:419-25.

35. López-Bermejo A, Chico-Julia B, Fernandez-Balsells M, et al. Serum Visfatin Increases With Progressive -Cell Deterioration. Diabetes 2006;55:2871-5. 